\title{
Research on the Influence of Economic Policy Uncertainty on the Supply Chain Finance
}

\author{
Li Wang ${ }^{1,2 a}$ Huimin Wang ${ }^{3,4 b}$ Jian Wang ${ }^{5,6 c}$ \\ ${ }^{1}$ School of Economics \& Management, Jiangsu University of Science \& Technology \\ ${ }^{2}$ CSSC Modern Logistics Research Center Zhenjiang, Jiangsu, China \\ ${ }^{3}$ School of Economics \& Management, Jiangsu University of Science \& Technology \\ ${ }^{4}$ Minsheng Securities Research Institute Zhenjiang, Jiangsu, China \\ ${ }^{5}$ School of Economics \& Management, Jiangsu University of Science \& Technology \\ ${ }^{6}$ CSSC Modern Logistics Research Center Zhenjiang, Jiangsu, China
}

\begin{abstract}
Based on the background of continuous increasing external economic uncertainty, this paper builds GARCH-MIDAS model to explore the volatility of copper price caused by global economic policy uncertainty in copper supply chain finance and analyzes the changes of refined copper supply and demand caused by this volatility. It is found that the increases of economic policy uncertainty will enhance the longterm volatility of copper. Moreover, the violent fluctuation of copper price caused by the impact of powerful economic policy uncertainty will weaken the demand confidence of refined copper market and lead to the phenomenon of oversupply. On the contrary, the moderate fluctuation of copper price due to the impact of weak economic policy uncertainty will boost the demand confidence of refined copper market and lead to the phenomenon of short supply.
\end{abstract}

\section{INTRODUCTION}

With uncertain events increase, such as trade barriers, Brexit and geopolitics, the economic growth of major global economies continued to slow down. The most intuitive impact of the grim economic situation is job losses. In 2019, more than 50 banks around the world cut 78000 employees. The turbulent economic environment will disrupt the structure of global value chain and bring adverse spillover effects. The negative impact of SinoU.S. trade frictions is more significant. According to IMF estimates, Sino-U.S. trade frictions will lead to the cumulative decline of global GDP to $0.8 \%$ in 2020 . In addition, covid-19 exacerbated the pessimism of investors, the global non-ferrous metal prices fell sharply. In the early stage of the outbreak, the market did not pay more attention to the coronavirus, which led to the LME copper falling from 6302 to 5567 dollars/ton for 14 consecutive trading days from January 14 to January 31 , down $11.7 \%$ year-on-year, among which the daily price fell more than $3 \%$ on January 27 . In the same period, LME aluminum fell 4.8\%, LME zinc fell $7.4 \%$, and LME nickel fell 7.4\%. Under the active control measures, policy support and rework after Spring Festival, LME copper price rebound mildly at a low level. The severity of the macroeconomic environment led to the high aversion in the market and the downward pressure on the economy continued to increase.

Supply chain finance refers to the optimization of supply chain processes, transactions management and the use of liquidity investment funds by using financing and risk mitigation measures and technologies. The core enterprises in the supply chain use their good reputation to obtain loans from banks for small and medium-sized enterprises. In addition, the core enterprises may pledge goods and extend the delivery period of loans to alleviate the financial difficulties of small and medium-sized enterprises. The deepening of globalization promotes the deep development of supply chain and the optimization of the internal structure of supply chain, but at the same time, the bad external environment is bound to bring risks to all participants in the supply chain. With the increasingly severe international situation, it is necessary to understand how the external economic environment affects the supply chain.

Stand on the current market environment, covid-19 is undoubtedly an important obstacle to supply chain imbalance. For the supply side, the medium-term production cannot be fully recovered, and the production capacity is seriously lagging behind. For the demand side, problems such as traffic jam and delayed resumption of work lead to the insufficient realization of consumption demand. Previous studies focused on the impact of economic policy uncertainty on the stock market and

a1 $\overline{484802359 @ q q . c o m{ }^{b} \text { liluomeng@ }}$ outlook.com * Corresponding author: cwangjscf@just.edu.cn 
commodities, but rarely explored the impact of uncertainty on the supply chain. This paper focuses on how economic policy uncertainty affects copper supply chain finance.

\section{LITERATURE REVIEW}

Macroeconomic uncertainty is an unpredictable conceptual term, which must be quantified. The widely used measurement is economic policy uncertainty, which takes authoritative newspapers as statistical samples to retrieve relevant articles contained key words such as "economic policy" and "uncertainty" and deletes unqualified articles according to requirements, then carries out standardization and other operations (Baker, 2015) [1]. It has been proved that the ability of EPU to predict the excess return of stock varies from country to industry (Phan, 2018), which is caused by the asymmetric impact of EPU [2]. Uncertainty has an anticyclical nature (Bachmann, 2013) [3], which inhibits investment decision-making and the negative impact will be more obvious during the recession (Kang, 2013) [4], so market participants should consider the impact of uncertainty when making investment decisions.

In supply chain finance, the research on uncertainty mainly focuses on internal uncertainty such as demand, supply and price, while the research on the impact of external uncertainty on supply chain finance is relatively scarce. Liu (2010) used the capital asset pricing model and net present value method to discuss the determination method of the optimal price, profit and value of the upstream and downstream enterprises in the supply chain under economic uncertainty [5]. In the context of the ongoing unresolved Brexit issue, Hendry (2019) analyzed the impact of Brexit uncertainty on the local food supply chain [6]. They believed that participants in the food supply chain should restructure their businesses if necessary to reduce the financial and trade dependence of EU to resist the risk of Brexit. The risk of Brexit urges the government to reduce food subsidies, then food prices rise, which disturbs the normal trading of food market. In extreme cases, there are violations of food safety standards, so government needs to prevent excessive supply and waste. In addition, Brexit will reshape the global supply chain structure (Nakamura, 2019) [7],

With the global spread of covid-19 and the continuous impact of multilateral trade disputes, IMF lowered its forecast of global economic growth on March 4, 2020 and predicted that the global economic growth rate in 2020 would be lower than $2.9 \%$ in 2019 . Uncertainty risk needs attention from all walks of life, including financial institutions such as enterprises and banks in the supply chain. When using or providing financial services, enterprises should enhance the professionalism of quantifying economic uncertainty, improve the ability to identify risks, and pay attention to controlling the proportion of capital investment. In addition, the enterprise management should perfect the corporate governance, optimize the ownership structure, establish a positive incentive and constraint mechanism, strengthen the internal risk control mechanism. This paper focuses on copper supply chain that can best reflect the macroeconomic environment and takes global economic policy uncertainty (GEPU) as an exogenous variable to empirically analyze the impact of GEPU on the copper supply chain.

\section{EMPIRICAL ANALYSIS}

\section{A. Data}

Supply chain finance shows advantages in reducing the financing pressure of small and medium-sized enterprises and stimulating the financing market. More enterprises join in the supply chain finance. Banks have also expanded and carried out the supply chain financial business to build a blue ocean of supply chain finance. Copper, as a necessary consumable for the development of global economy, is consumed a lot. There is no alternative metal product. The demand for copper can directly reflect the state of economic activities. With the change of real economy, the price of copper will fluctuate. This paper chooses copper supply chain finance as research sample and takes LME copper futures as research object. The sample range is from January 2003 to January 2020. This sample has 4273 observations. The returns of copper futures are calculated by logarithm and difference of LME copper futures price. GEPU is an index weighted by GDP and economic policy uncertainty (EPU) of 21 countries. In this paper, ARMA (p, q) model is used to process exogenous variables. The optimal order of $p$ and $q$ is determined by AIC criterion and the residual of the model is squared as the volatility of exogenous variables.

$$
X_{t}=\sum_{i=1}^{p} \varphi_{i} X_{t-i}+\sum_{i=1}^{q} \theta_{i} \varepsilon_{t-i}+\varepsilon_{t}
$$

In addition, this paper takes the surplus/gap of refined copper as another exogenous variable, which is an important indicator to measure the supply and demand of copper supply chain finance, then analyzes the volatility of surplus/gap of refined copper on copper prices. As the global refined copper surplus/gap data is not fully disclosed, the sample range selected for this indicator is from August 2004 to November 2019 and it has 183 observations.

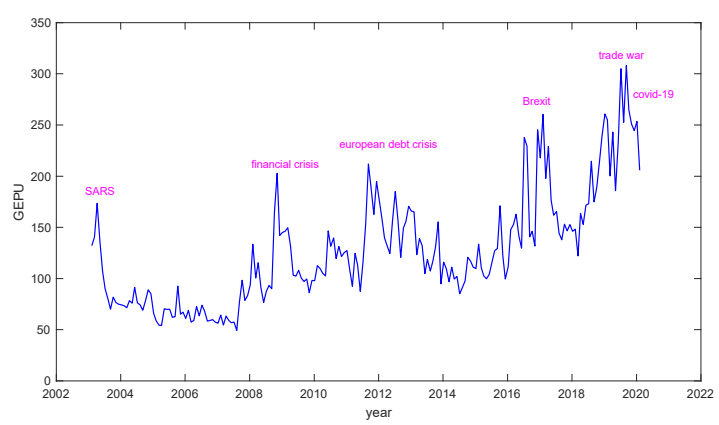

Figure 1 Global economic policy uncertainty index (GEPU) 


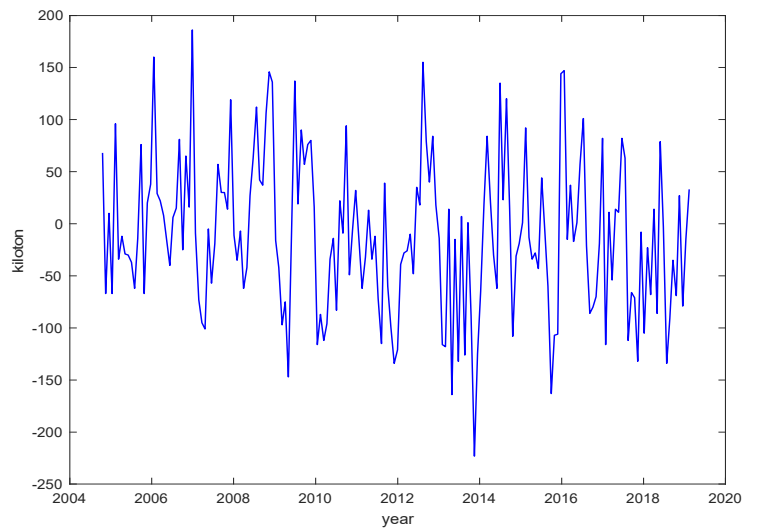

Figure 2 Global refined copper surplus / gap

\section{B. Model Selection}

In this paper, GARCH-MIDAS model is selected to analyze the volatility of copper futures prices by GEPU and how this volatility will affect supply and demand by global copper surplus/gap. GARCHMIDAS model decomposes the asset price volatility series into shortterm and long-term volatility. The short-term volatility is characterized by high-frequency data such as asset daily volatility, and the long-term volatility $l_{t}$ is characterized by low-frequency data such as asset monthly volatility. The specific structure is as follows:

$$
r_{i, t}=\mu+\sqrt{l_{t} \times g_{i, t}} \xi_{i, t} \quad \forall i=1, \ldots, N_{t}
$$

$r_{i, t}$ represents the logarithmic returns of assets on the trading day. $\mu$ is the conditional mean. A month has $N_{t}$ days, which obeys the independent and identical distribution with the mean value of 0 and the variance of 1. The conditional variance of $r_{i, t}$ is equal to the product of short-term component $g_{i, t}$ and long-term component $l_{t}$ : $\sigma_{i, t}^{2}=l_{t} g_{i, t}$.

The short-term components $g_{i, t}$ are satisfied with GARCH $(1,1)$ process

$$
g_{i, t}=(1-\alpha-\beta)+\alpha \frac{\left(r_{i-1, t}-u\right)^{2}}{l_{t}}+\beta g_{i-1, t}
$$

$\alpha>0, \beta>0, \alpha+\beta<1$. Smoothing realized volatility estimates of long-term components by MIDAS regression:

$$
\begin{gathered}
l_{t}=m+\theta \sum_{k=1}^{K} \varphi_{k}\left(\omega_{1}, \omega_{2}\right) X_{t-k} \\
R V_{t}=\sum_{i=1}^{N_{t}} r_{i, t}^{2}
\end{gathered}
$$

$k$ is the number of lag periods, $K$ represents the maximum number of lag periods of low-frequency variables, determined by the maximum likelihood function.

The above weight is called $\beta$ weights:

$$
\varphi_{k}\left(\omega_{1}, \omega_{2}\right)=\frac{(k / K)^{w_{1}-1}\left(1-\frac{k}{K}\right)^{w_{2}-1}}{\sum_{j=1}^{K}(j / K)^{w_{1}-1}\left(1-\frac{j}{K}\right)^{w_{2}-1}}
$$

$\sum_{k=1}^{K} \varphi_{k}\left(\omega_{1}, \omega_{2}\right)=1$. Generally, $\omega_{1}$ set as 1 , as long as $\omega_{2}$ is estimated. The parameter space of the model is $\Theta=\left\{\mu, \alpha, \beta, m, \theta, \omega_{1}, \omega_{2}\right\}$.

In addition, this paper uses four loss function values to compare volatility prediction accuracy of GARCHMIDAS, GARCH, EGARCH and GJR model. They are RMSE (root mean square error of conditional variance), RMAE (mean absolute error of conditional variance), MSPE (pure mean square error of conditional variance) and MAPE (mean absolute percentage error of conditional variance).

$$
\begin{gathered}
R M S E=\sqrt{\frac{1}{T} \sum_{t=1}^{T}\left(\sigma_{t+1}^{2}-E_{t}\left(\sigma_{t+1}^{2}\right)\right)^{2}} \\
R M A E=\sqrt{\frac{1}{T} \sum_{t=1}^{T}\left|\sigma_{t+1}^{2}-E_{t}\left(\sigma_{t+1}^{2}\right)\right|} \\
M S P E=\frac{1}{T} \sum_{t=1}^{T}\left(\sigma_{t+1}-E_{t}\left(\sigma_{t+1}\right)\right)^{2} \\
M A P E=\frac{1}{T} \sum_{t=1}^{T}\left|\sigma_{t+1}-E_{t}\left(\sigma_{t+1}\right)\right| \\
D M=\frac{\frac{1}{d}}{\sqrt{\operatorname{var}\left(d_{t}\right)}} \sim N(0,1) \\
d_{t}=e_{A, t}^{2}-e_{B, t}^{2}
\end{gathered}
$$

$\sigma_{t+1}^{2}$ is the conditional variance, i.e. the daily realized volatility. $E_{t}\left(\sigma_{t+1}^{2}\right)$ is the predicted value of the conditional variance, and $\mathrm{T}$ is the number of observations. $e_{A, t}, e_{B, t}$ are the prediction errors of model A and model $\mathrm{B}$ respectively. $\bar{d}$ is the mean value of time series $d_{t}$ and $\operatorname{var}\left(d_{t}\right)$ is the variance of time series $d_{t}$.

\section{Empirical results}

\begin{tabular}{|c|c|c|c|}
\hline & GARCH-MIDAS-RV & GARCH-MIDAS-GEPU & $\begin{array}{l}\text { GARCH-MIDAS- } \\
\text { Surplus/gap }\end{array}$ \\
\hline$\mu$ & $\begin{array}{l}1.292 \mathrm{e}-04 \\
(2.2195 \mathrm{e}-04)\end{array}$ & $\begin{array}{l}1.2085 \mathrm{e}-04 \\
(2.1951 \mathrm{e}-04)\end{array}$ & $\begin{array}{l}-1.2213 \mathrm{e}-05 \\
(2.3553 \mathrm{e}-04) \\
\end{array}$ \\
\hline$\alpha$ & $\begin{array}{l}0.05283^{* * *} \\
(0.0056)\end{array}$ & $\begin{array}{l}0.04473^{* * * *} \\
(0.0045)\end{array}$ & $\begin{array}{l}0.0484^{* * *} \\
(0.0047)\end{array}$ \\
\hline$\beta$ & $\begin{array}{l}0.9274^{* * * *} \\
(0.0094)\end{array}$ & $\begin{array}{l}0.9487^{* * *} \\
(0.0051)\end{array}$ & $\begin{array}{l}0.9456^{* * *} \\
(0.0054)\end{array}$ \\
\hline$\theta$ & $\begin{array}{r}0.1663^{* * * *} \\
(0.0151)\end{array}$ & $\begin{array}{r}0.0020^{* * * *} \\
(0.0006)\end{array}$ & $\begin{array}{l}0.0742^{*} \\
(0.0409)\end{array}$ \\
\hline$\omega$ & $\begin{array}{l}1.5102^{* *} \\
(0.7230)\end{array}$ & $\begin{array}{c}13.859^{* * *} \\
(4.629)\end{array}$ & $\begin{array}{l}23.043 \\
(21.638)\end{array}$ \\
\hline$m$ & $\begin{array}{c}8.5642 \mathrm{e}-03^{* * * *} \\
(1.3801 \mathrm{e}-03)\end{array}$ & $\begin{array}{c}1.8113 \mathrm{e}-04^{* * *} \\
(0.3339 \mathrm{e}-04)\end{array}$ & $\begin{array}{l}-8.3621^{* * *} \\
(0.1956)\end{array}$ \\
\hline $\mathbf{L L}$ & 10482.1 & 10486.6 & 9250.77 \\
\hline AIC & -20952.1 & -20961.2 & -18489.5 \\
\hline RMSE & $5.710 \mathrm{e}-04$ & $5.698 \mathrm{e}-04$ & - \\
\hline
\end{tabular}

In this section, GARCH-MIDAS model is used to empirically analyze the impact of GEPU on the volatility of copper price and how the supply and demand of refined copper to change in the copper supply chain caused by this volatility. In this paper, the supply and demand of refined copper is represented by the global surplus/gap of refined copper. Surplus means that the supply of refined copper is over demand, and gap means that the supply of refined copper is under demand.

Table 1: parameter estimation of GARCH-MIDAS model 


\begin{tabular}{|l|l|l|l|}
\hline MSPE & $5.4732 \mathrm{e}-04$ & $5.4207 \mathrm{e}-04$ & - \\
\hline MAPE & 0.0180 & 0.0179 & - \\
\hline
\end{tabular}

Note: * indicates that the estimated value is significant at the level of $10 \% ; * *$ indicates that the estimated value is significant at the level of $5 \%$; ** indicates that the estimated value is significant at the level of $1 \%$. The value in brackets is the standard error.

In the parameter estimation of GARCH-MIDAS-RV model, the intercept term $\mu$ is not significant at the level of $5 \%$, the parameter value $\omega$ is significant at the level of $5 \%$, and the other parameters are significant at the level of $1 \%$. Parameter value $\omega$ is greater than $1, \varphi_{k}(\omega)$ will decrease with the increase of lag period $k$, which shows that the forward yield has less effect on the copper fluctuation in the current period.

In the parameter estimation of GARCH-MIDASGEPU model, the long-term mean value of copper logarithm yield $\mu$ is not significant, which is consistent with the empirical results of Zheng (2014), and the influence of long-term mean $\mu$ can be eliminated. The parameters $\alpha+\beta<1$ meet the constraints, which indicates that the long-term fluctuation of copper gradually converges to a stable value and copper return has a significant GARCH effect. The parameter value $\theta$ is positive, indicating that the long-term volatility of copper will increase with the increase of economic policy uncertainty. The parameter value $\omega$ is 13.859 , which is significant at the level of $1 \%$, indicating that the economic policy uncertainty has a great impact on the long-term volatility of LME copper, so when the economic policy uncertainty index is abnormally large, the response of long-term volatility of LME copper will be very sensitive. The global economy has experienced the impact of strong uncertainties such as the financial crisis in 2008, the debt crisis in 2011 and the Brexit in 2017. The long-term volatility of copper in these three stages is extremely fierce (Fig. 4). The title of "copper doctor" deserves it! In contrast, the parameter $\omega$ in the realized volatility model is only 1.5102 , and the longterm volatility of copper is relatively less affected by the realized volatility impact (Fig. 3). In 2020, the risk of new coronavirus has refreshed various data in the financial market. From Fig. 4, we can see that the longterm volatility of copper after January 2020 still has a relatively obvious growth trend. In addition, the AIC information criterion of the calculation model can avoid the over fitting phenomenon of the model. The AIC value of GARCH-MIDAS-GEPU model is smaller than that of GARCH-MIDAS-RV model, indicating that the former data fitting is better. Moreover, the RMSE, MSPE and MAPE of GARCH-MIDAS-GPEU model are smaller than those of GARCH-MIDAS-RV model, so the fitting effect of GARCH-MIDAS-GEPU model is better. Therefore, adding exogenous variable GEPU can improve the prediction accuracy of the model.

In the parameter estimation of GARCH-MIDASsurplus/gap model, the parameters $\alpha$ and $\beta$ are significant at the level of $1 \%$ and the sum of two is less than 1 , which meets the constraints. The parameter value $\theta$ is 0.0742 , which is positive and significant at the $10 \%$ level. The sharp fluctuation of copper price caused by the impact of strong economic uncertainty will weaken the demand confidence of refined copper market and lead to the phenomenon of oversupply. On the contrary, the moderate fluctuation of copper price due to the impact of weak economic uncertainty will boost the demand confidence of refined copper market and lead to the phenomenon of short supply. In 2008, the financial crisis led to a surplus of 376000 tons of refined copper. In 2014, the uncertainty of economic policy was relatively weak, the demand side of copper supply chain was more active, namely, refined copper consumption was strong. In addition, the world's major miners are facing huge pressure of rising costs, which made the growth rate of copper supply is lower than the market expectation, resulting in the phenomenon of short supply. In that year, there was a total gap of 459000 tons of refined copper, a record low. On June 23, 2016, the UK announced the referendum on Brexit. The copper market suffered a huge setback and the demand side tightened, leading to a serious surplus of refined copper in the second half of 2016.

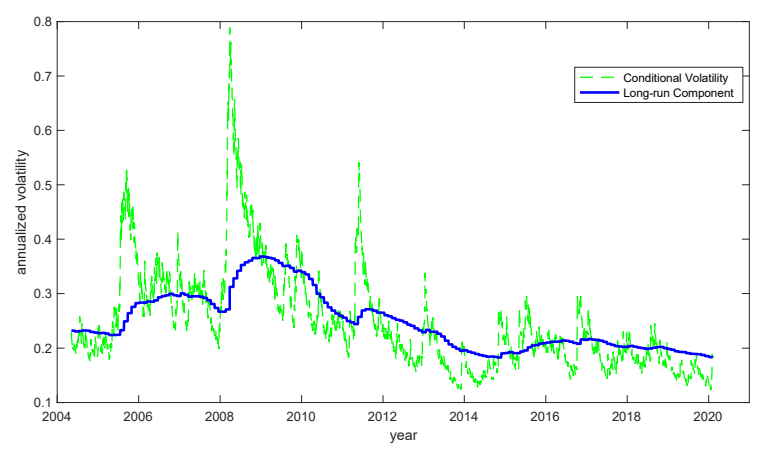

Figure 3 decomposition of copper volatility based on realized volatility $\mathrm{RV}$

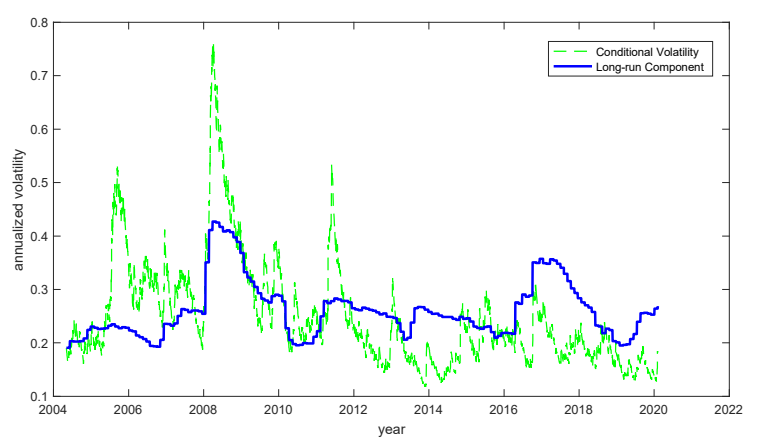

Figure 4 decomposition of copper volatility based on GEPU

\section{Out-Sample forecast}

In this section, first $60 \%$ of the sample data is used for in-sample estimation of GARCH-MIDAS-GEPU model. The in-sample estimation is basically similar to the parameter estimation of the whole sample. The parameter $\omega$ is significant at the level of $10 \%$ and other parameters are significant at the level of $1 \%$. $\alpha$ and $\beta$ meet constraint conditions of the parameters. Then last $40 \%$ of the sample data is used to estimate the out-sample prediction error of GARCH-MIDAS, GARCH, EGARCH and GJR traditional volatility models. The smaller the loss function is, the more accurate the prediction of the model is. By comparing 
the loss function of each model in Table 2, it is found that the loss function of GARCH-MIDAS-GEPU model is the smallest. It can be considered that the prediction accuracy of GARCH-MIDAS model can be improved by adding the exogenous variable GEPU. In this paper, GARCH-MIDAS-GEPU is used as the benchmark model and DM test is carried out for each model. The DM statistics of GARCH-MIDAS-RV, GARCH, EGARCH and GJR models are negative, indicating that the prediction performance of these comparison models is weaker than that of the benchmark model.

Table 2: In-Sample forecast estimation in GARCHMIDAS-GEPU model

\begin{tabular}{|l|l|l|l|l|}
\hline$\mu$ & $\alpha$ & $\beta$ & $\theta$ & $\omega$ \\
\hline $\begin{array}{l}6.2562 \mathrm{e}-04^{* * *} \\
(3.7724 \mathrm{e}-04)\end{array}$ & $\begin{array}{l}0.0606^{* * *} \\
(0.0087)\end{array}$ & $\begin{array}{l}0.9211^{* * *} \\
(0.0142)\end{array}$ & $\begin{array}{c}0.1614^{* * *} \\
(0.0313)\end{array}$ & $\begin{array}{c}1.7111^{*} \\
(1.2508)\end{array}$ \\
\hline$m$ & AIC & LLF & & \\
\hline $\begin{array}{c}0.0110^{* * *} \\
(0.0036)\end{array}$ & -10502.5 & 5257.27 & & \\
\hline
\end{tabular}

Table 3: Out-sample prediction error of comparison model

\begin{tabular}{|l|l|l|l|l|}
\hline & RMSE & RMAE & MSPE & MAPE \\
\hline $\begin{array}{l}\text { GARCH-MIDAS- } \\
\text { RV }\end{array}$ & $2.6676 \mathrm{e}-04$ & 0.0120 & $2.6891 \mathrm{e}-04$ & 0.0136 \\
\hline $\begin{array}{l}\text { GARCH-MIDAS- } \\
\text { GEPU }\end{array}$ & $2.0717 \mathrm{e}-04$ & 0.0119 & $2.6075 \mathrm{e}-04$ & 0.0133 \\
\hline GARCH & $6.8610 \mathrm{e}-04$ & 0.0179 & $5.1007 \mathrm{e}-04$ & 0.0178 \\
\hline EGARCH & $4.0506-04$ & 0.0156 & $4.0506 \mathrm{e}-04$ & 0.0156 \\
\hline GJR & $6.7288 \mathrm{e}-04$ & 0.0164 & $4.1335 \mathrm{e}-04$ & 0.0157 \\
\hline
\end{tabular}

Table 4: DM Test

\begin{tabular}{|l|l|l|l|l|}
\hline & GARCH-MIDAS-RV & GARCH & EGARCH & GJR \\
\hline DM & -0.2513 & -0.1309 & -0.1311 & -0.1309 \\
\hline
\end{tabular}

Note: GARCH-MIDAS-GEPU model is the benchmark model for DM inspection, and the figures in the table are model DM.

\section{CONCLUSIONS}

This paper explores the fluctuation of copper future price in the copper supply chain finance caused by global economic policy and analyzes the changes of refined copper supply and demand in the copper supply chain caused by this fluctuation. Firstly, this paper reviews the study of economic policy uncertainty (EPU), which covers policy and macroeconomic uncertainty information. After that, according to the existing literature review, it is found that the application of economic uncertainty in supply chain finance is relatively scarce, so the significance of this paper is put forward. This paper constructs comparative models of GARCH-MIDAS-RV and GARCH-MIDAS-GEPU and finds that the impact of economic policy uncertainty on the long-term volatility of copper is more intense. Refined copper is an important raw material in the middle and lower reaches of the copper supply chain. In this paper, the global surplus/gap of refined copper is expressed as a supply-demand relationship, which is an important indicator of the copper supply chain. The research shows that the sharp fluctuation of copper price caused by the impact of strong economic policy uncertainty will weaken the demand confidence of refined copper market and lead to the phenomenon of oversupply. On the contrary, the moderate fluctuation of copper price due to the impact of weak economic uncertainty will boost the demand confidence of refined copper market and lead to the phenomenon of short supply. Finally, four loss functions and DM test are used to compare the prediction accuracy of the traditional GARCH, EGARCH, GJR model with that of GARCHMIDAS-GEPU model. It is confirmed that the prediction performance of GARCH-MIDAS will be improved by adding the exogenous variable economic policy uncertainty. At present, the economic environment is more and more severe. Enterprises carrying out copper supply chain financial projects should pay more attention to economic uncertainty and improve their ability to resist the risk of economic uncertainty.

\section{Acknowledgment}

This work is financial supported by National Natural Science Foundation of China (Grand No.71802100, 71873111), Humanities and Social Science project of Ministry of Education of China (Grant No.18YJAZH024), Social Science project of Jiangsu education department (No.2018SJA1087)

\section{REFERENCES}

1. S. R. Baker, N. Bloom, S. J. Davis, "Measuring economic policy uncertainty," NBER Working Paper, No. 21633.

2. D. H. B. Phan, S. S. Sharma, V. T. Tran, "Can economic policy uncertainty predict stock returns? Global evidence," Journal of International Financial Markets, Institutions \& Money, vol. 55, 2018, pp. 134-150.

3. R. Bachmann, S. Elstner and E. R. Sims, "Uncertainty and economic activity: Evidence from business survey data," American Economic Journal: Macroeconomics, vol. 5, 2013, pp. 217-249.

4. W. Kang, K. Lee and R. A. Ratti, "Economic policy uncertainty and firm-level investment," MPRA paper, No51277, 2013, pp. 1-32.

5. Z. Liu, J. M. Cruz, "Supply chain networks with corporate financial risks and trade credit under economic uncertainty," International journal of production economics, vol. 137, 2010, pp. 55-67.

6. L. C. Hendry, M. Stevenson, J. MacBryde, P. Ball, M. Sayed and L. Liu, "Local food supply chain resilience to constitutional change: the Brexit effect," International Journal of Operations \& Production Management, vol. 39, 2019, pp. 429453.

7. K. Nakamura, T. Yamada and K. Tan, "The impact of Brexit on designing a material-based global supply chain network for Asian manufacturers," Management of Environmental Quality, vol. 30, 2019, pp. 980-1000.

8. T. G. Zheng, Y. H. Shang, "Measurement and prediction of stock market volatility based on 
macro fundamentals," World Economy, vol. 12, 2014, pp. 118-139. 